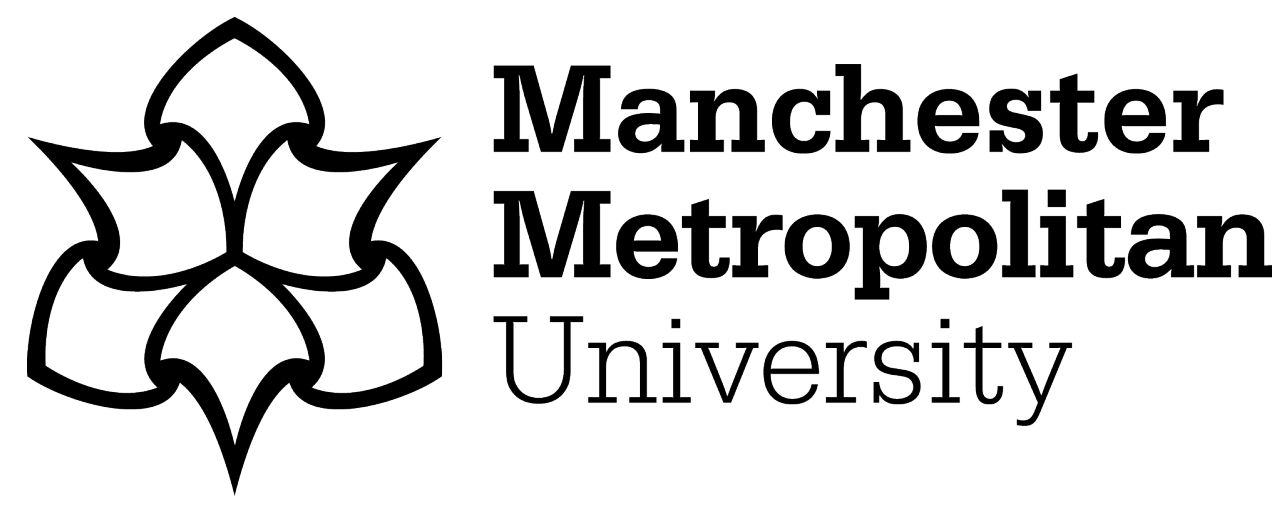

Rajbhandari, Sujan, Haas, Harald, Xie, Enyuan, McKendry, Jonathan JD, Herrnsdorf, Johannes, Gu, Erdan, Dawson, Martin D, O’Brien, Dominic, Chun, Hyunchae, Faulkner, Grahame, Cameron, Katherine, Jalajakumari, Aravind VN, Henderson, Robert, Tsonev, Dobroslav, ljaz, Muhammad ORCID logoORCID: https://orcid.org/0000-0002-0050-9435 and Chen, Zhe (2015) High-Speed Integrated Visible Light Communication System: Device Constraints and Design Considerations. IEEE Journal on Selected Areas in Communications, 33 (9). pp. 1750-1757. ISSN 0733-8716

Downloaded from: https://e-space.mmu.ac.uk/625200/

Version: Accepted Version

Publisher: Institute of Electrical and Electronics Engineers (IEEE)

DOI: https://doi.org/10.1109/jsac.2015.2432551

Please cite the published version 


\title{
High-Speed Integrated Visible Light Communication System: Device Constraints and Design Considerations
}

\author{
Sujan Rajbhandari, Member, IEEE, Hyunchae Chun, Grahame Faulkner, Katherine Cameron, \\ Aravind V. N. Jalajakumari, Robert Henderson, Dobroslav Tsonev, Member, IEEE, Muhammad Ijaz, Zhe Chen, \\ Harald Haas, Member, IEEE, Enyuan Xie, Jonathan J. D. McKendry, Member, IEEE, Johannes Herrnsdorf, \\ Erdan Gu, Martin D. Dawson, Fellow, IEEE, and Dominic O’Brien, Member, IEEE
}

\begin{abstract}
Visible light communications (VLC) has the potential to play a major part in future smart home and next generation communication networks. There is significant ongoing work to increase the achievable data rates using VLC, to standardize it and integrate it within existing network infrastructures. The future of VLC systems depends on the ability to fabricate low cost transceiver components and to realize the promise of high data rates. This paper reports the design and fabrication of integrated transmitter and receiver components. The transmitter uses a two dimensional individually addressable array of micro light emitting diodes ( $\mu$ LEDs) and the receiver uses an integrated photodiode array fabricated in a CMOS technology. A preliminary result of a MIMO system implementation operating at a data rate of 1 Gbps is demonstrated. This paper also highlights the challenges in achieving highly parallel data communication along with the possible bottlenecks in integrated approaches.
\end{abstract}

Index Terms-Visible light communications, optical communication system design, multiple input multiple output, optical wireless communications, link budget analysis, integrated optical system design.

\section{INTRODUCTION}

$\mathbf{T}$ HERE has been significant research interest in visible light communications (VLC) in the last decade. This is largely due to the possibility of using general illumination lightemitting diode (LED) devices for data communications. The LED is expected to dominate the illumination market by 2020

Manuscript received May 10, 2014; revised November 13, 2014; accepted April 13, 2015. Date of publication May 14, 2015; date of current version August 17, 2015. This work was supported by the UK Engineering and Physical Sciences Research Council (EPSRC) under grant EP/K00042X/1.

S. Rajbhandari, H. Chun, G. Faulkner, and D. O'Brien are with the Department of Engineering Science, University of Oxford, Oxford OX1 3PJ, U.K. (e-mail: sujan.rajbhandari@eng.ox.ac.uk; hyunchae.chun@eng.ox.ac.uk; grahame.faulkner@eng.ox.ac.uk; dominic.obrien@eng.ox.ac.uk).

K. Cameron, A. V. N. Jalajakumari, and R. Henderson are with CMOS Sensors \& Systems Group, The University of Edinburgh, Edinburgh EH9 3JL, U.K. (e-mail: K.Cameron@ed.ac.uk; A.Venugopalan@ed.ac.uk; Robert. Henderson@ed.ac.uk).

D. Tsonev, M. Ijaz, Z. Chen, and H. Haas are with the Institute for Digital Communications, Li-FI R\&D Centre, The University of Edinburgh, Edinburgh EH9 3JL, U.K. (e-mail: d.tsonev@ed.ac.uk; m.ijaz@ed.ac.uk; z.chen@ed.ac.uk; h.haas@ed.ac.uk).

E. Xie, J. J. D. McKendry, J. Herrnsdorf, E. Gu, and M. D. Dawson are with the Institute of Photonics, University of Strathclyde, Glasgow G1 1XQ, U.K. (e-mail: enyuan.xie@strath.ac.uk; jonathan.mckendry@strath.ac.uk; johannes. herrnsdorf@strath.ac.uk; erdan.gu@strath.ac.uk; m.dawson@strath.ac.uk).

Color versions of one or more of the figures in this paper are available online at http://ieeexplore.ieee.org.

Digital Object Identifier 10.1109/JSAC.2015.2432551
[1], and can be used for high speed data communications [2]. VLC using LEDs offers many advantages including license free operation, high spatial diversity and innate security.

Two methods of generating white light are commercially popular: a) an RGB method in which light from red, green, and blue LEDs are mixed, resulting in a white color and b) a phosphor conversion method, in which a yellow phosphor absorbs a portion of the blue light emitted by a Gallium Nitride $(\mathrm{GaN})$ LED and re-emits a broad yellow spectrum, which when mixed with the blue wavelength results in a white color. Commercially available illuminations LEDs usually use the phosphor conversion method due to its low cost.

Phosphor based LEDs, however, have low communication bandwidths (a few $\mathrm{MHz}$ ) due to the long photoluminescence lifetimes of the phosphor [3]. Typically a narrowband short pass optical filter is used at the receiver to the reject the slow yellow component of the received light. The bandwidth of the blue LED, on the other hand, is limited to $20-30 \mathrm{MHz}$ [3] which is a possible bottleneck for high speed data communications. Recently, it has been shown that GaN-based blue-emitting micro-LEDs ( $\mu$ LEDs) can offer optical modulation bandwidths in excess of $400 \mathrm{MHz}$ [4]. Error-free data transmission up to $1 \mathrm{Gbps}$ and $3 \mathrm{Gbps}$ using these $\mu$ LEDs was demonstrated using on-off keying (OOK) and orthogonal frequency division multiplexing (OFDM) [5], respectively. Although these experiments were carried out over a short distance due to the limited optical power available, these results nonetheless demonstrate the potential of the GaN based $\mu$ LEDs to offer high-speed communication. The ultimate solution for VLC may be to use an array of these smaller, less powerful $\mu$ LEDs as building blocks [6].

Work to explore the potential of these devices is ongoing under the Engineering and Physical Sciences Research Council (EPSRC) "ultra-parallel visible light communications (UPVLC)' project. The project aims to develop a high speed, highly parallel VLC system that can offer Gbps data rates. This paper focuses on communication using blue $\mu$ LEDs. Other work in the project focuses on combining light from such devices with high bandwidth color converters [7] to create white light. Ultimately the combination of such color converters and large arrays of $\mu$ LEDs offers the potential to combine very high data rate communication and illumination in a single transmitter. 
The focus in this paper is on the design of the transmitter and the receiver components and on possible approaches for achieving a high-speed data link. The target of the first UPVLC demonstrator is to realize a $1 \mathrm{Gbps}$ unidirectional data link over a $1 \mathrm{~m}$ distance at error rate of $10^{-6}$. Links offering similar data rates have been achieved using low bandwidth commercial white LEDs [8]-[10], albeit at a bit error rate (BER) floor of $\sim 10^{-3}$. In our case relatively modest data rates were chosen as a target for this initial demonstration, as the focus is to develope integrated components suitable for scaling to much higher data rates in subsequent demonstrators.

Although $\mu$ LEDs have higher bandwidth than commercial devices, arrays of devices are required to support the target data rates due to the limited optical power available from individual devices. Three approaches are investigated here;

a) A ganging approach: all $\mu$ LEDs in the array carry the same data

b) A multiple input multiple output (MIMO) approach: each $\mu$ LED in an array carries an independent data stream

c) A hybrid approach, which combines a) and b)

The MIMO system can offer a linear increment in data rate with number of transmitters but requires channel separation using imaging/non-imaging optics and MIMO data decoding algorithms.

The ganging approach offers simplicity in design as simple receiver circuitry can be used but does not offer a linear increase in data rate with the number of transmitters. The hybrid approach combines the advantage of both approaches. The system components can also implement different transmission schemes including spatial modulation [11], and optically generated modulation [12]. All these approaches will be implemented within the project.

The performance of a VLC system is a function of the constraints of the transmitter (the LED diameter, optical power, and bandwidth) and receiver (photodiodes area and bandwidth/area/sensitivity relationship). In this paper, we report a design approach that includes these constraints and allows the overall optimum configuration to be determined. To our knowledge, this is the first report of such a method.

The paper is organized as follows: Section II gives an overview of the system being developed. Section III details the approach taken to select the device parameters. Optical and electronic system designs are outlined in Sections IV and $\mathrm{V}$, respectively. The experimental results are presented in Section VI. Finally, the conclusions and future work are given in Section VII.

\section{System DESCRIPTION}

Fig. 1 shows a conceptual block diagram of the system under development. The transmitter consists of a 2-D array of $\mu$ LEDs operating at a wavelength of $450 \mathrm{~nm}$. These $\mu$ LEDs are driven by complementary metal-oxide-semiconductor (CMOS) drivers. The $\mu$ LEDs are Lambertian sources with a divergence angle of 120 degrees (full-angle). Transmitter optics reduces the divergence angle and hence reduces the path loss. Imaging optics at the receiver maps the $\mu$ LED array to the photodiode (PD) array. For minimum cross-talk between the MIMO

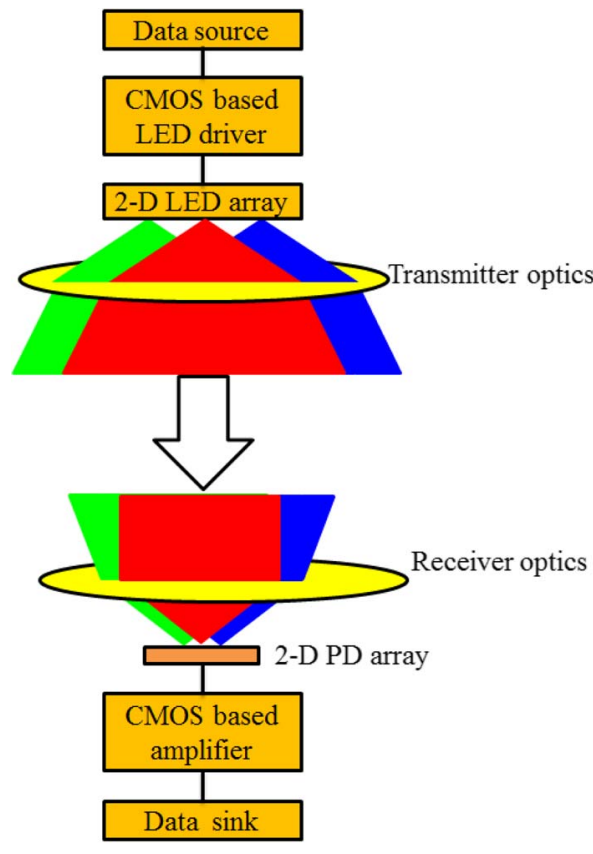

Fig. 1. A conceptual block diagram of the VLC system (different colours are used for illustration only. The central wavelength of $\mu$ LEDs considered in this design is $450 \mathrm{~nm}$ ).

channels in an imaging system, the PDs and the $\mu$ LEDs pitch sizes must be matched. The ganging scheme does not require imaging optics, and a diffuser can be used after the transmitter optics to provide the desired FOV (details of optics designs are given in the following sections).

At the receiver, the PDs photocurrents are converted to a voltage using transimpedance amplifiers (TIA) and are either summed (for ganging) or processed separately (for MIMO). The MIMO receiver can overcome any crosstalk by estimating the channel H-matrix and recover data using MIMO data decoding algorithms. The details of the MIMO decoding algorithm can be found in [13], [14].

\section{Design Methods: System Constraints AND PARAMETER SELECTIONS}

Both $\mu$ LEDs and PDs have constraints which need to be considered in the system design. By incorporating the power penalty for multilevel PAM ( $L$-PAM), $\mu$ LED constraints (bandwidth-power relationship) and PD constraints (area-bandwidth relationship), it is feasible to optimize the device parameters and modulation level to achieve the target data rate with a minimum number of transmitter and receiver elements. The details of the devices selection and link budget analysis are given in the following sections. For simplicity, only a line-of-sight (LOS) link is considered in the design, and only L-PAM is considered. However, the design method has flexibility to incorporate equalization and other complex modulation schemes.

\section{A. Selection of Device Parameters}

In order to establish the optimum $\mu$ LED parameters (i.e., size, power and bandwidth) for the targeted data rate, the 


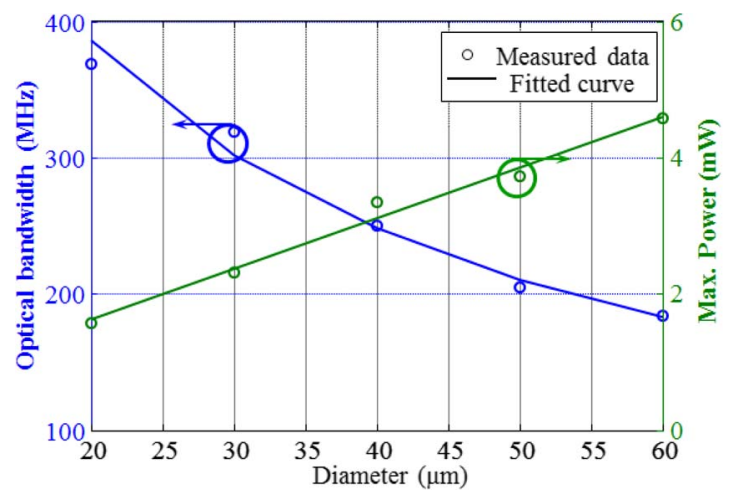

Fig. 2. The relationship among the optical bandwidth, optical power and $\mu \mathrm{LED}$ diameter. The measured data and fitted curves are also shown.

interdependency between power and bandwidth of $\mu \mathrm{LED}$ is investigated. Fig. 2 shows the measured optical bandwidth and the maximum optical power for blue $\mu$ LEDs with different diameters. It can be seen that there is an approximately inverse linear relationship between the maximum optical power and bandwidth, both of which are a function of the $\mu$ LED diameter. For the blue $\mu$ LEDs reported in [4], the following equations approximately describe the relationship between the $\mu$ LEDs diameter $d_{L E D}$, maximum optical power $P_{\text {opt }}$ and optical bandwidth $B_{o p t}$ (valid for $\mu$ LEDs with a diameter of 20-80 $\mu \mathrm{m}$ ):

$$
\begin{aligned}
P_{\text {opt }}[\mathrm{mW}] & =\frac{1013}{B_{\text {opt }}[\mathrm{MHz}]}-0.985 ; \\
P_{\text {opt }}[\mathrm{mW}] & =0.0743 d_{L E D}[\mu m]+0.132
\end{aligned}
$$

The bandwidth and power requirements for a link depend on: a) the target data rate, b) the required order of the multilevel modulation scheme and c) the system configuration (ganging, MIMO). The MIMO scheme requires lower bandwidth $\mu$ LEDs as the data rate per MIMO channel can be made significantly lower than the aggregate data rate. For the ganging scheme, the bandwidth requirement can be reduced by increasing the number of levels in a multilevel modulation scheme, at the cost of a higher optical power requirement. Hence, the optimum bandwidth and power requirements for the MIMO and the ganging configurations are different and can only be established by taking into account the device constraints and the modulation scheme.

There are also device constraints at the receiver. For a CMOS $\mathrm{PD}$, the relationship between the PD capacitance $\mathcal{C}_{\mathrm{PD}}$ and area AreapD can be expressed as [15]:

$$
\mathcal{C}_{\mathrm{PD}}=\frac{\text { AreapD }_{J}}{\left(1+\frac{V}{V_{B}}\right)^{M_{J}}}+\frac{\mathcal{P} \mathcal{C}_{\mathrm{JSW}}}{\left(1+\frac{V}{V_{B}}\right)^{M_{\mathrm{JSW}}}}
$$

where $\mathcal{C}_{J}$ is junction capacitance, $\mathcal{C}_{\text {JSW }}$ is sidewall junction capacitance, $M_{J}$ is junction grading coefficient, $M_{J S W}$ is sidewall junction grading coefficient, $V_{B}$ is junction potential and $\mathcal{P}$ is the perimeter. In a shallow junction photodiode, as is considered here, the area component is dominant leading to $\mathcal{C} \propto$ AreapD.

There are a number of different TIA designs that can be used. In [16] chapter 4, it was shown that for a fixed power

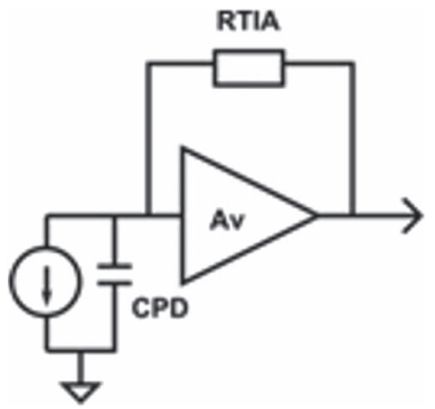

Fig. 3. The schematic of shunt-shunt feedback TIA topology. CPD, the capacitance of the photodiode, is assumed to be the dominant input capacitance.

consumption the shunt-shunt feedback topology (Fig. 3) will have better noise performance than alternatives. As the noise performance is critical, this design is used. In this case the bandwidth $B_{\text {TIA }}$ is given by [16]:

$$
B_{\mathrm{TIA}}=\frac{A_{V}}{2 \pi R_{T I A} C_{\mathrm{PD}}}
$$

where $A_{V}$ is the voltage gain of the amplifier used in the TIA and $R_{T I A}$ is the feedback resistance. Therefore:

$$
B_{\mathrm{TIA}} \approx \frac{A_{V}\left(1+\frac{\mathrm{V}}{P_{B}}\right)^{M_{J}}}{\operatorname{AreapD}_{J}\left(2 \pi R_{T I A}\right)}=\frac{K}{\text { AreapD }}
$$

where $P_{B}$ is junction potential and $K$ is a constant whose value is dependent on process parameters and TIA gain/structure.

It can be seen that bandwidth is inversely proportional to area. As the received power is a function of the receiver collection area, this relationship must be taken into account.

\section{B. SNR Analysis of L-PAM Considering Transmitter and Receiver Constraints}

PAM is one of the most popular modulation schemes in VLC systems. PAM is attractive because of the simplicity in the transmitter and receiver design.

Multilevel modulation requires higher received optical power to achieve the same bit rate and error performance as binary modulation, but offer a reduction in the bandwidth requirement.

In this paper, designs using $L$-PAM is considered. To achieve a data rate of $R_{b}$ in an additive white Gaussian noise (AWGN) channel, the bandwidth $B$ and optical power penalty $P_{\mathrm{pb}}$ for $L$-PAM to achieve a desired error probability relative to OOK is given by [17], [18]:

$$
\begin{aligned}
B & =\frac{1}{M} \\
P_{\mathrm{pb}} & =\frac{(L-1)}{\sqrt{M}} ;
\end{aligned}
$$

where $L=2^{M}$ and $M$ is a positive integer.

The error probability is a function of available electrical SNR which is defined as [18], [19]:

$$
\mathrm{SNR}_{\text {available }}=\frac{\left(R P_{r}\right)^{2}}{\sigma_{a}^{2}}=\frac{\left(R H(0) P_{T}\right)^{2}}{N_{0} B}=K_{1} \frac{P_{T}^{2} \mathrm{Area}_{\mathrm{PD}}^{2}}{B} ;
$$


where $R$ is the PD responsivity, $P_{T}$ and $P_{r}$ are the average transmitted and received optical power respectively, $\sigma_{a}^{2}$ is the amplifier noise variance, $N_{0}$ is the double-sided noise powerspectral, $K_{1}$ is a constant and $H(0)$ is the channel DC gain. This is a function of propagation distance $d$, incident angle $\Phi$ and order of Lambertian emission $m$, and is given by:

$$
H(0)=\frac{\operatorname{AreapD}_{\mathrm{PD}}(m+1)}{2 \pi d^{2}} \cos ^{m}(\Phi) .
$$

Therefore, the available SNR, considering the transmitter and receiver constraints in (1) and (5) is given by:

$$
\mathrm{SNR}_{\text {available }} \propto\left\{\begin{array}{ll}
B^{-3} & \text { considering only(5) } \\
B^{-5} & \text { considering (1) and (5) }
\end{array} ;\right.
$$

Considering the receiver system constraints in (5), the available SNR for $L$-PAM normalized to that of OOK for a given optical power is given by:

$$
S N R_{L-\mathrm{PAM}}=M^{3} .
$$

As indicated from (7), $L$-PAM requires an additional SNR of $(L-1)^{2} / M$ to achieve the same bit error rate (BER) as OOK. Assuming the PD area can be adjusted to match the bandwidth requirement for $L$-PAM, the receiver system can offer an SNR improvement of $M^{3}$. Hence $L$-PAM offers an SNR gain in comparison to OOK if the available SNR gain is higher than the additional SNR requirements i.e.

$$
M^{3}>\frac{(L-1)^{2}}{M} .
$$

This condition is satisfied for $M<5$ and a maximum SNR gain $\left[M^{3}-\left\{(L-1)^{2} / M\right\}\right]$ of $2.5 \mathrm{~dB}$ is obtained for 4-PAM. This indicates that 4-PAM requires the minimum transmitted power to achieve the target data rate and BER as long as the PD area can be varied to match the required system bandwidth.

In obtaining (13), the transmitter device constraints are not included in the analysis i.e. it is assumed that the transmitted power is fixed. In our design, it is feasible to manufacture a $\mu$ LED that can match the system requirements. Hence, the $\mu$ LED's bandwidth and power constraints can also be included within the link budget analysis. By incorporating transmitter constraints (1) in (9), it can be shown that $L$-PAM offers an SNR gain if:

$$
M^{5}>\frac{(L-1)^{2}}{M} .
$$

This condition is satisfied for $M<10$ and a maximum SNR gain of $12.6 \mathrm{~dB}$ is obtained for 16-PAM.

The analysis here is limited to PAM based modulation without an equalizer. The equalization and complex modulation schemes like OFDM offers further improvement in system performance. The analysis of power requirement for equalization and OFDM is beyond the scope of the paper. Interested reader can refer to [20]-[22]. However, the demonstrator allows these schemes to be implemented, so that detailed experimental comparisons can be made.

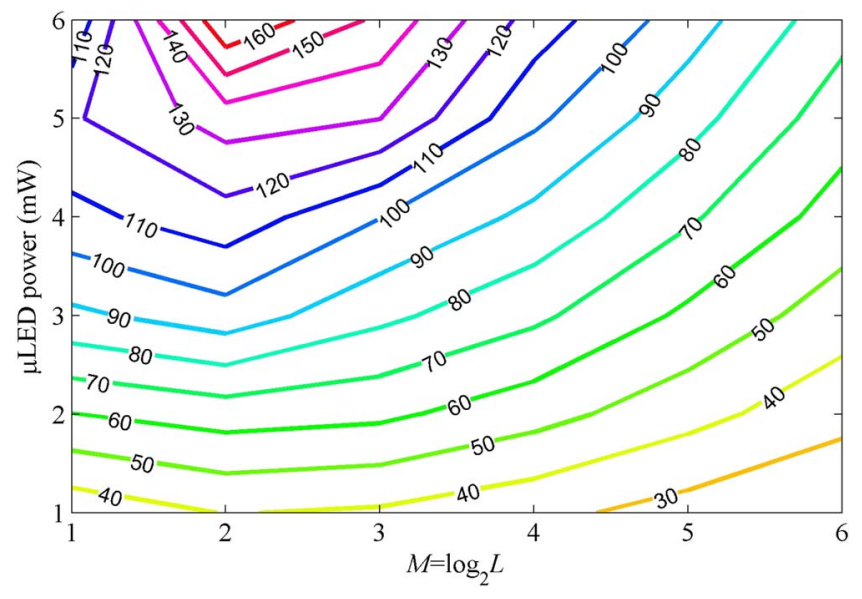

\begin{tabular}{|c|c|c|c|}
\hline \multirow{2}{*}{ Parameters } & & \multicolumn{2}{|c|}{ Values } \\
\hline & & Ganging & MIMO \\
\hline \multicolumn{2}{|l|}{ Data rate (Gbps) } & $>1$ & $>1$ \\
\hline \multicolumn{2}{|l|}{ Link Length (m) } & $>1$ & $>1$ \\
\hline \multicolumn{2}{|c|}{ Number of parallel channel } & 1 & 4 \\
\hline \multirow{6}{*}{$\mu$ LEDs } & Array size & $6 \times 6$ & $6 \times 6$ \\
\hline & Wavelength (nm) & 450 & 450 \\
\hline & Diameter $(\mu \mathrm{m})$ & 24 & 39 \\
\hline & Optical power $(\mathrm{mW})$ & 2 & 3 \\
\hline & Bandwidth (MHz) & 175 & 125 \\
\hline & Pitch size $(\mathrm{mm})$ & 0.3 & 1.5 \\
\hline Transmitter optics & $\begin{array}{l}\text { Divergence angle } \\
\text { (full) }\end{array}$ & $10^{\circ}$ & $7.5^{\circ}$ \\
\hline \multirow{2}{*}{ Receiver Optics } & FOV (full angle) & $8^{\circ}$ & $3^{\circ}$ \\
\hline & Gain (maximum) & 296 & 1182 \\
\hline \multirow{5}{*}{$\begin{array}{l}\text { Photodiode } \\
\text { (APD) }\end{array}$} & Array size & $3 \times 3$ & $3 \times 3$ \\
\hline & Width $(\mu \mathrm{m})$ & 200 & 200 \\
\hline & Pitch length $(\mu \mathrm{m})$ & 240 & 240 \\
\hline & $\begin{array}{l}\text { Responsivity @ } \\
450 \mathrm{~nm}(\mathrm{~A} / \mathrm{W})\end{array}$ & 2.41 & 2.41 \\
\hline & Bandwidth (MHz) & 175 & 175 \\
\hline
\end{tabular}

Fig. 4. A contour plot of the maximum achievable data rates (Mbps) using $L$-PAM for different $\mu$ LED powers.

TABLE I

DESIGN PARAMETERS OF VLC DEMONSTRATOR-I

\section{Approaches to Achieve Higher Data Rate: Ganging and MIMO}

Fig. 4 shows the maximum achievable data rates at a $1 \mathrm{~m}$ link distance using a single $\mu \mathrm{LED}$ and a single PD. The link budget analysis assumes a target BER of $10^{-6}$, a $10 \mathrm{~dB}$ link margin and a divergence/FOV of 5 degrees (half angle). As predicted from (12), 4-PAM requires the minimum power to achieve the desired data rate. Though the lower power $\mu$ LEDs have higher bandwidth, there is not enough link margin to support higher data rates.

In order to achieve a target data rate of $1 \mathrm{Gbps}$, either ganging or MIMO approaches are required. The optimum device parameters for these approaches are established based on the analysis detailed in previous sections and summarised in Table I. 


\section{Demonstration Optics Design}

Imaging MIMO systems are reported in [11], [23]. The work reported in [11] provides the theoretical capacity of such a system based on the assumptions that the receiver image is an orthographic projection of the transmitted image. An optical design for an integrated angle diversity imaging receiver is also reported in [24]. In this paper we focus on designs that use commercially available optical components for both the MIMO and ganging schemes. In order to achieve a common optical design the constraints for both schemes need to be considered. Both imaging and non-imaging optical concentrators can be used in the ganging scheme. Though the non-imaging concentrator can provide optical 'gain' close to the theoretical limit set by the constant radiance theorem, the channel H-matrix is ill-conditioned in a non-imaging MIMO system [25]. The $\mathrm{H}$-matrix must be of full rank in order to successfully separate the MIMO channels at the receiver. To achieve this in an imaging MIMO system, the image of more than one source should not fall entirely into the same receiver and hence the imaging MIMO system must satisfy the following condition:

$$
\frac{p}{f} \leq \frac{s}{d}
$$

where $s$ is the source spacing, $p$ is the PD width, $f$ is the focal length of the receiver optics system and $d$ is the propagation length. The ratio $(p / f)$ also governs the receiver FOV of the imaging system. The maximum detector area for the target data rate is established from the area-bandwidth relationship (see analysis in Section III). Hence, a higher FOV can be achieved only by reducing the focal length. However, due to physical constraints, it is not feasible to design a lens system with larger input aperture but smaller focal length (note that the optical 'gain' depends on the input aperture and high gain is desirable). Hence there is a trade-off between the optical "gain" and the FOV.

The FOV for an individual PD in imaging system is limited. In order to increase the FOV, the number of PDs is made significantly greater than the minimum requirement for a pointto-point link. For a larger FOV, the desired PD number can be in the order of thousand [26]. Increasing the number of receiver elements also increases the receiver complexity and the cost. With the integrated approach taken here, it is believed that the system is scalable to accommodate a large number of PDs.

The specification for demonstrator-I is a $3 \times 3$ array of PDs with dimensions of $200 \times 200 \mu \mathrm{m}^{2}$ on a $240 \mu \mathrm{m}$ pitch (details of these PD are given in the following sections). This gives a full FOV of 3 degrees for a lens system with $f=11 \mathrm{~mm}$. A larger PD array system will be fabricated in the next phase of the project once the initial assumptions and designs are tested and verified. Increasing the number of PDs will increase the FOV and hence will reduce the need to align the transmitter and receiver.

Considering the constraints imposed by (14), the minimum desirable distance between the transmitter elements is $44 \mathrm{~mm}$. In order to limit the chip size, the transmitter optics has been designed in such a way that it generates a virtual image of transmitters with the desired pitch.

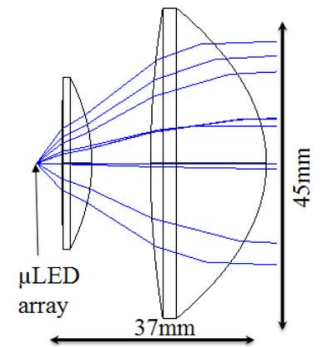

Transmitter optical system

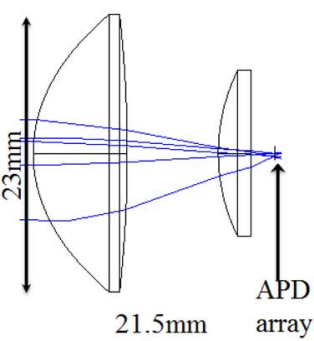

Receiver optical system

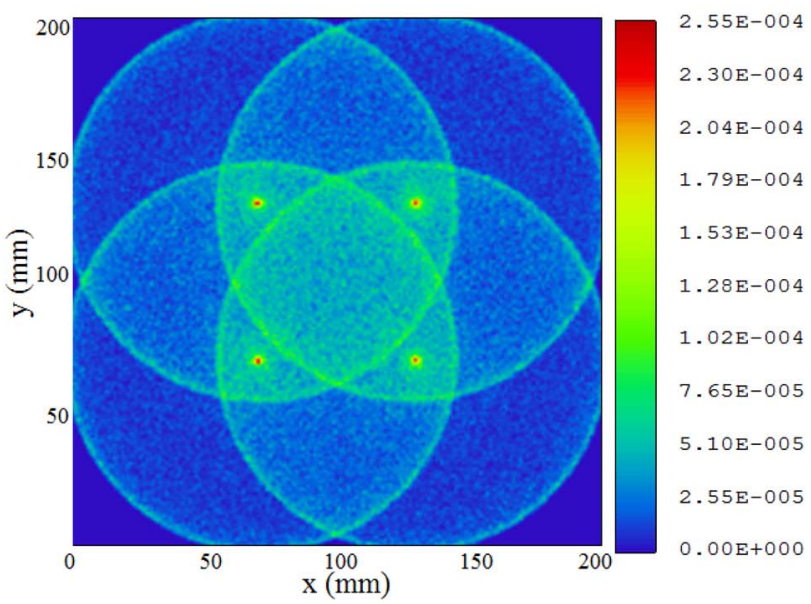

(b)

Fig. 5. a) Schematic of optical system and snapshot of the opto-mechanical assemlies. b) simulated optical irradiance $\left(\mathrm{W} / \mathrm{cm}^{2}\right)$ in a $200 \times 200 \mathrm{~mm}^{2}$ receiver plane at $1 \mathrm{~m}$ distance from the transmitter.

The transmitter optics also limits the divergence angle of the $\mu$ LED so that the geometric loss can be minimised. The Lambertian emission of the $\mu$ LED means that a high numerical aperture optical system is required, and a suitable system was designed and optimised using ray-tracing software. The final optical designs and their mechanical assemblies for the MIMO system are shown in Fig. 5(a). Fig. 5(b) shows the optical irradiance at a $1 \mathrm{~m}$ distance on a $200 \times 200 \mathrm{~mm}^{2}$ plane. The transmitter is designed to offer a full divergence angle of 7.5 degrees and an overlapping area of 4.5 degrees, where all the MIMO channels will operate. Note that a MIMO system can operate only in the central overlapping area where all four MIMO channels overlap. The receiver has full FOV of 3 degrees. For the ganging system, an appropriate holographic diffuser is used to create a transmitter beam with a divergence angle of 10 degrees. 


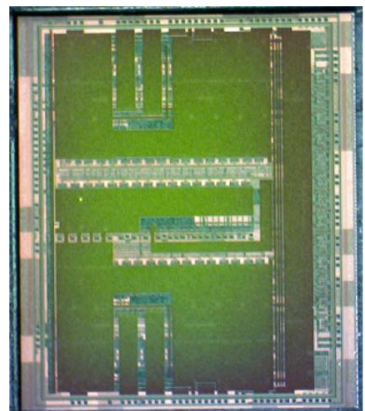

(a)

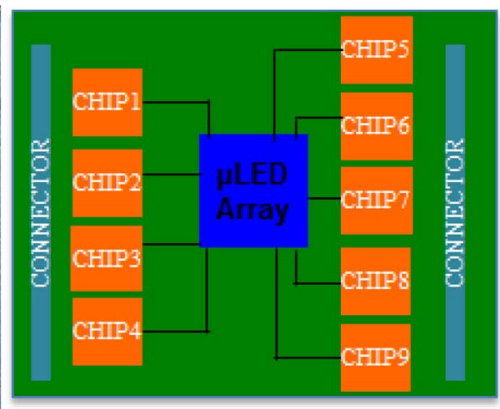

(b)
Fig. 6. $\mu$ LED driver a) fabricated die and b) transmitter PCB block diagram.

\section{Electronics Design}

\section{A. Transmitter Subsystem}

This consists of $\mu \mathrm{LED}$ driver chips, the $\mu \mathrm{LED}$ array and associated printed circuit board (PCB). The $\mu$ LED driver chip is implemented in an Austria Micro Systems 0.18 $\mu \mathrm{m}$ CMOS process, building on previous designs [4]. Circuit specifications were derived from system level simulations and calculations mentioned in previous sections. Each driver chip consists of 4 independent $\mu$ LED drivers, interfacing and configuration circuitry. The chip also has an internal buffer/de-serializer to support ganging/MIMO operation. Chip configuration can be performed through the serial interface provided. An n-channel metal-oxide semiconductor (NMOS) transistor based circuit is used to drive the $\mu \mathrm{LEDs}$, because of the higher carrier mobility and lower area requirement compared with p-channel MOS (pMOS) transistors. Each driver channel can sink an LED drive current up to $255 \mathrm{~mA}$, and is designed to operate at a bandwidth of up to $250 \mathrm{MHz}$.

Fig. 6 shows driver chip block diagram and photograph of the fabricated chip. Nine driver chips and a $6 \times 6 \mu \mathrm{LED}$ array are interconnected through the transmitter PCB (Fig. 6(b)), which also mounts the transmitter optical assembly. Separate data interface PCBs can be attached to the transmitter PCB for ganging and MIMO operation.

\section{B. $\mu L E D s$}

Both ganging and MIMO $\mu$ LED devices consist of an array of individually-addressable $6 \times 6$ elements in a flip-chip configuration. In order to be compatible with the NMOS-based CMOS driver, each $\mu$ LED element in these devices has an individual n-type contact, whereas all elements share a common p-contact. The disk-shaped $\mu$ LED element has a diameter of $24 \mu \mathrm{m}$ for the ganging and $39 \mu \mathrm{m}$ for the MIMO device. As shown in Fig. 7(a), the ganging $\mu$ LED array has a uniform pitch of $300 \mu \mathrm{m}$. In order to match the transmitter pitch to that of the receiver, the MIMO array is arranged such that the elements are grouped into $2 \times 2$ clusters with a pitch of $69 \mu \mathrm{m}$ between two adjacent $\mu$ LED elements. There is a separation between the end-to-end elements of $1500 \mu \mathrm{m}$ (see Fig. 7(b)). The four closely clustered $\mu$ LED elements also provide the ability of operating in a hybrid mode, as described earlier.

These devices are fabricated on commercial blue InGaN/ GaN LED wafers grown on c-plane (0001) sapphire sub-
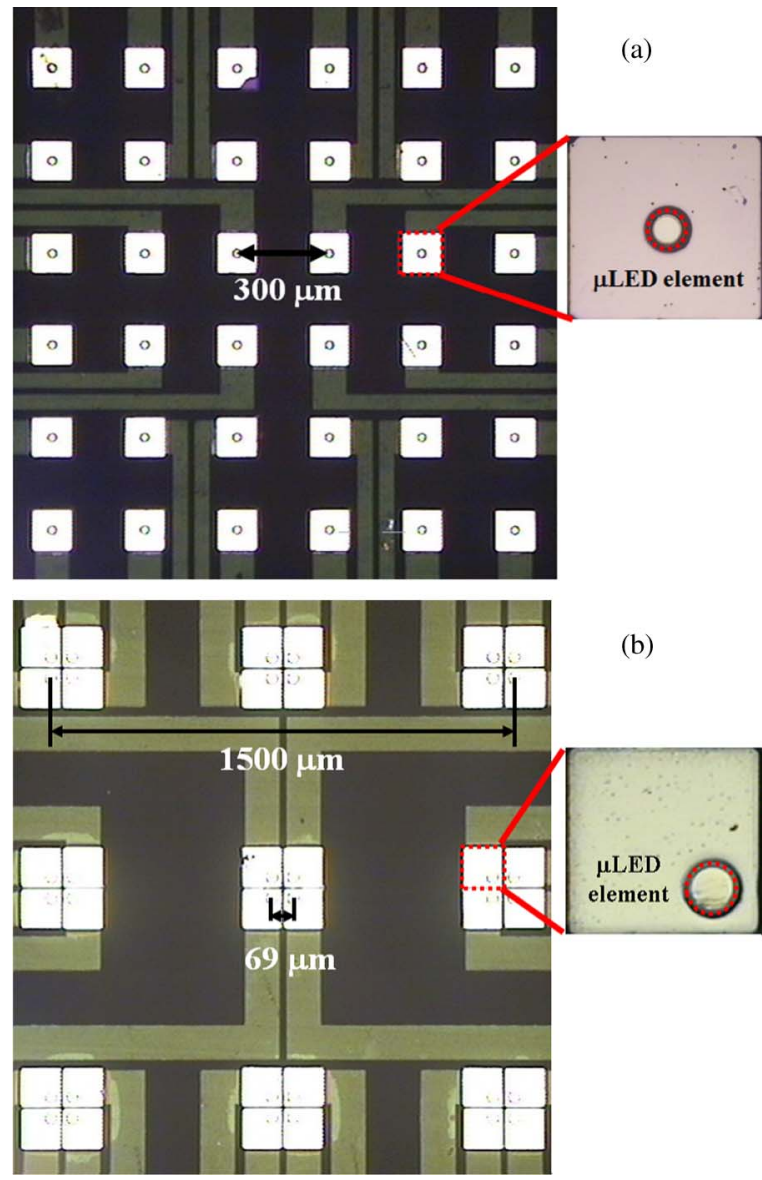

Fig. 7. a) Images of a) $\mu$ LED array of the ganging device and b) $\mu$ LED array of the MIMO device. High-magnification images for typical $\mu$ LED elements are also shown.

strates with a $450 \mathrm{~nm}$ peak emission wavelength and $20 \mathrm{~nm}$ full width at half maximum. In a first step, $6 \times 6$ mesa structures are deeply etched down to the sapphire substrate by $\mathrm{Cl}_{2}$-based inductively coupled plasma etching. Then a second etch step defines the $\mu$ LED elements on each mesa. These steps allow each LED element to be addressed by its own n-contact appropriate for driving with an NMOS-based CMOS driver. A thermally annealed $\mathrm{Pd}$ layer with over $50 \%$ reflectance at $450 \mathrm{~nm}$ is used as the metal contact to p-type GaN. A metal bilayer of Ti/Au serves as the metal contact to n-type $\mathrm{GaN}$ and metal tracks. After the $\mu$ LED fabrication, the ganging and MIMO devices are bonded to the backside of a 132-pin package using Norland optical adhesive and then wire bonded. This backside arrangement reduces the separation between light emission surface (sapphire surface) and the optical system. Finally, the bonded device is contacted with a copper heat sink.

\section{Receiver}

Modelling indicated that in order to obtain sufficient receiver sensitivity, an APD based receiver is required, with a typical input referred noise density of $10 \mathrm{pA} / \sqrt{ } \mathrm{Hz}$. This is challenging compared with some designs. As a comparison, [27] reports referred noise for each channel of $29.9 \mathrm{pA} / \sqrt{ } / \mathrm{Hz}$. In [28], Shimotori et al. showed that the APD structure shown in Fig. 8 can be made in a $0.18 \mu \mathrm{m}$ process and have a responsivity to $405 \mathrm{~nm}$ light of $2.61 \mathrm{~A} / \mathrm{W}$ at a reverse bias voltage of $9.1 \mathrm{~V}$. 


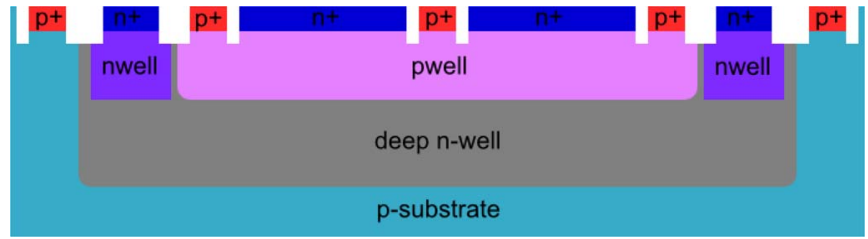

Fig. 8. A potential APD structure as reported in [28].

The measured bandwidth of the structure was $300 \mathrm{MHz}$ which also fits in with the specification in Table I. The APD in [28] is $20 \mu \mathrm{m} \times 20 \mu \mathrm{m}$. Our requirement is for $200 \mu \mathrm{m} \times 200 \mu \mathrm{m}$. In addition to the bandwidth limitation imposed on the system by the capacitance of the APD (5), the intrinsic bandwidth of the photodiode can limit performance. A full description of the intrinsic bandwidth can be found in chapter 3 of [29] but it is related to the transit time of optically generated carriers across the APD. In [30], it was shown that through this effect the bandwidth reduces with increasing size of APD. This led to the hypothesis that it is better to build the APD out of a $10 \times 10$ array of $20 \mu \mathrm{m} \times 20 \mu \mathrm{m}$ structures, whose outputs can be summed together by connecting the structures in parallel, in order to meet the bandwidth requirements. This method was also used in the paper recently published by Ray et al. [31].

The dominant source of noise will come from the TIA connected to the APD. In chapter 7 of [32], it is shown that if the input stage to the amplifier in a shunt-shunt feedback topology is a CMOS inverter then the total input-referred noise is:

$$
\begin{aligned}
I_{\text {in }}=4 k_{B} T B\left[\frac{1}{R_{T I A}}+2 \frac{\Gamma_{F}}{g_{m p}+g_{m n}}\right. & \frac{\left(2 \pi C_{P D} B\right)^{2}}{3} \\
+ & \left.\frac{\Gamma_{F}}{R_{T I A}^{2}\left(g_{m p}+g_{m n}\right)}\right]
\end{aligned}
$$

where $k_{B}$ is Boltzmann s constant, $T$ is temperature, $R_{\text {TIA }}$ is the feedback resistor, $C_{\mathrm{PD}}$ is the capacitance of the PD, $\Gamma_{F}$ is the process dependent gamma factor and $g_{m p}$ and $g_{m n}$ are the transconductances of the input transistors. From the equation it can be seen that to reduce noise, the $g_{m}$ of the input transistors must be increased which will increase the power consumption of the chip. At this point the power budget is not a limiting factor, but for future revisions there will have to be a trade-off between the number of channels and the power consumption of each channel which will in turn depend on the avalanche gain of the APDs. This may alter some of the design decisions.

\section{EXPERIMENTAL RESULTS}

The current status of the demonstration is that most of the components are manufactured and individually tested. The next step is to integrate the individual components to full a scale system demonstration. The measured electrical-to-electrical $-3 \mathrm{~dB}$ bandwidths of ganging and MIMO $\mu$ LED devices are shown in Fig. 9. The target bandwidths of 175 and $125 \mathrm{MHz}$ for ganging and MIMO devices are met at bias currents of $55 \mathrm{~mA}$ and $100 \mathrm{~mA}$, respectively. The measured optical powers at these currents are 2 and $3.5 \mathrm{~mW}$, respectively. The transmitter and receiver designs were also verified by measuring the intensity profile and channel gain for the MIMO system at a distance of $1 \mathrm{~m}$ from the transmitter that match the designed profile.

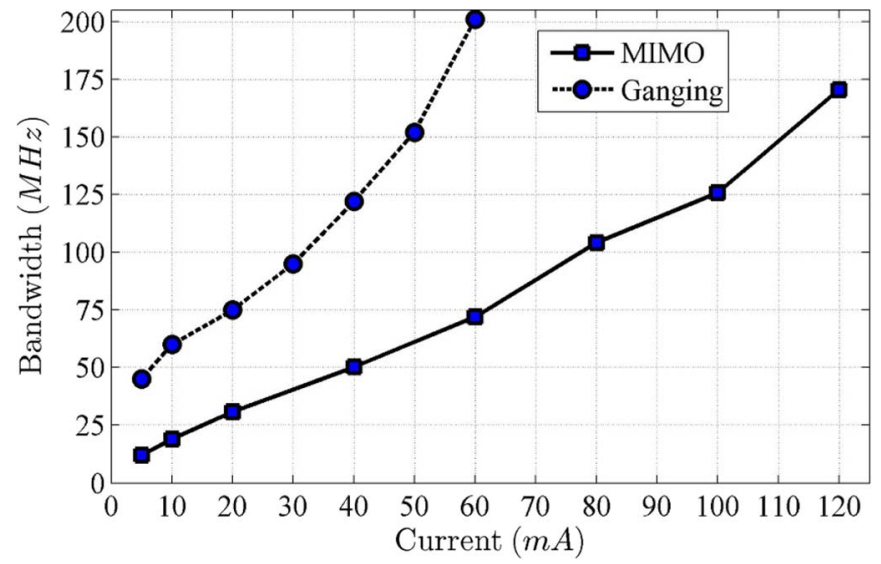

Fig. 9. Measured electrical-to-electrical bandwidths against the bias current of ganging and MIMO devices.

In order to demonstrate the potential of the design, a MIMO communication link using the available components was tested. At the transmitter an array of four $\mu$ LEDs was used, each channel driven using an arbitrary waveform generator. The $3 \times 3$ APD receiver array described earlier is used. Issues with the CMOS process limited the available bandwidth of each APD to $22 \mathrm{MHz}$. Due to the limited capability of the implementation; an OOK modulation scheme with decision feedback equaliser (DFE) was used in the experiment.

The four $\mu$ LEDs were driven by four independent pseudo random binary sequences of length $2^{14}-1$. The LEDs were biased at a DC current of $50 \mathrm{~mA}$ and an AC swing of $3.5 \mathrm{Vpp}$. The received signals from four APDs were then captured simultaneously using an Oscilloscope (MSO7104B), and further signal processing was done offline. The received data sequences were then compared with the transmitted sequence to estimate the bit error rate (BER).

Fig. 10 shows the measured BERs of different channels against data rate for the imaging MIMO system. The BER is estimated using a sequence of at least $40 \times 10^{5}$ bits per channel. The achievable data rates above the forward error correction (FEC) threshold of $2 \times 10^{-3}$ [32] for channels 1-4 are 270, 290, 325, and 240 Mbps, respectively. The aggregate BERs against the data rates for MIMO system is also shown in Fig. 10. The MIMO system achieves a data rate of $1.070 \mathrm{Gbps}$, which correspond to a net rate of $\sim 1$ Gbps after an FEC overhead reduction of 7\% [33].

The subsystems required to be fully tested the MIMO and ganging schemes, using both OFDM and PAM are almost complete, including a modified APD array that should reach the required design bandwidth. System optimisation and testing will be carried out once all these are available.

\section{CONCLUSiON AND Future WORK}

This paper reports the detailed design of a demonstration VLC link. It shows that the optimum system design must take into account the particular characteristics of an emitter and receiver technology, and in this case there is an optimum modulation scheme that maximizes achievable data rate.

The simulation and analysis also shows that there is significant challenge in achieving highly parallel data links with a 


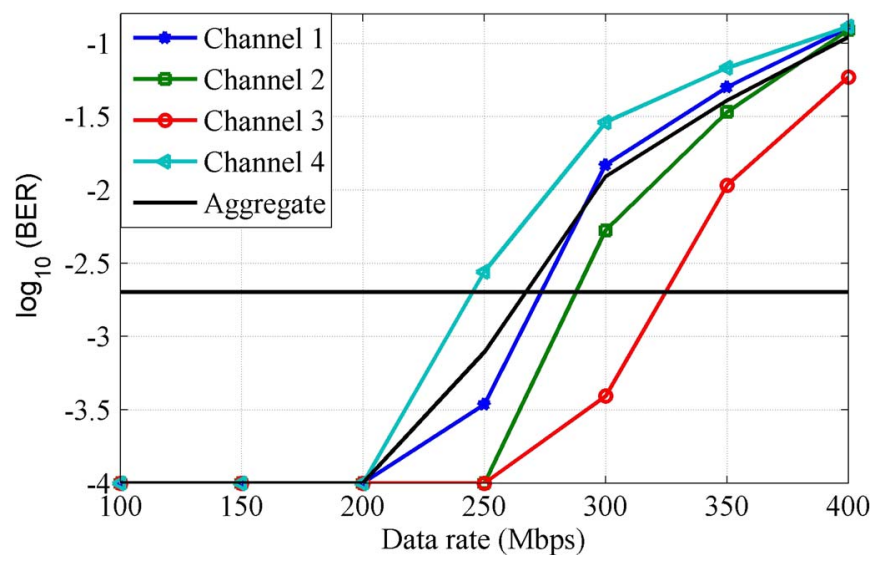

Fig. 10. Data rates against the BER for individual channel and aggregate MIMO system. Note that MIMO data rate is divided by 4 for the clarity of the figure.

larger FOV. The number of receiver elements increases rapidly with the increased FOV. The desire of high optical "gain" at the receiver also makes it challenging to miniaturize such systems for future integration in hand-held devices.

The paper also reports preliminary experimental results. Using a MIMO configuration, an aggregate data rate of $\sim 1$ Gbps after FEC overhead reduction is achieved. Future work includes complete system test and characterisation. A number of modulation schemes including OFDM and PAM will be tested and experimental comparisons will be made. The next phase of the project will address the challenges of scaling the system to higher data rates and wider field of view. This is likely to require large numbers of emitter and receiver channels, and the issues of addressing such devices in a scalable way, as well as the necessary signal processing for schemes such as OFDM. These challenges will be fully considered in the future iteration of the designs.

\section{ACKNOWLEDGMENT}

The authors gratefully acknowledge support by the UK Engineering and Physical Sciences Research Council (EPSRC). Associated data, including the ray tracing files and data for the figures can be found at DOI 10.5287/bodleian:xp68kg80s.

\section{REFERENCES}

[1] Lighting the Way: Perspectives on the Global Lighting Market, McKinsey \& Company, New York, NY, USA, 2012.

[2] L. Grobe et al., "High-speed visible light communication systems," IEEE Commun. Mag., vol. 51, no. 12, pp. 60-66, Dec. 2013.

[3] H. L. Minh et al., "100-Mb/s NRZ visible light communications using a postequalized white LED," IEEE Photon. Technol. Lett., vol. 21, no. 15, pp. 1063-1065, Aug. 2009.

[4] J. J. D. McKendry et al., "Visible-light communications using a CMOScontrolled micro-light- emitting-diode array," J. Lightw. Technol., vol. 30, no. 1, pp. 61-67, Jan. 2012.

[5] D. Tsonev et al., "A 3-Gb/s single-LED OFDM-based wireless VLC link using a Gallium Nitride $\mu$ LED," IEEE Photon. Technol. Lett., vol. 26, no. 7, pp. 637-640, Apr. 2014.

[6] A. Jovicic, L. Junyi, and T. Richardson, "Visible light communication: Opportunities, challenges and the path to market," IEEE Commun. Mag., vol. 51, no. 12, pp. 26-32, Dec. 2013.

[7] H. Chun et al., "Visible light communication using a blue GaN $\mu$ LED and fluorescent polymer color converter," IEEE Photon. Technol. Lett., vol. 26, no. 20, pp. 2035-2038, Oct. 2014.

[8] F.-M. Wu et al., "1.1-Gb/s white-LED-based visible light communication employing carrier-less amplitude and phase modulation," IEEE Photon. Technol. Lett., vol. 24, no. 19, pp. 1730-1732, Oct. 2012.
[9] A. M. Khalid, G. Cossu, R. Corsini, P. Choudhury, and E. Ciaramella, "1-Gb/s transmission over a phosphorescent white LED by using rateadaptive discrete multitone modulation," IEEE Photon. J., vol. 4, no. 5, pp. 1465-1473, Oct. 2012.

[10] C. Kottke, J. Hilt, K. Habel, J. Vucic, and K. D. Langer, "1.25 Gbit/s visible light WDM link based on DMT modulation of a single RGB LED luminary," in Proc. 38th ECOC, 2012, pp. 1-3.

[11] S. Hranilovic and F. R. Kschischang, "A pixelated MIMO wireless optical communication system," IEEE J. Sel. Topics Quantum Electron., vol. 12, no. 4, pp. 859-874, Jul./Aug. 2006.

[12] J. F. Li et al., "Superposed pulse amplitude modulation for visible light communication," Opt. Exp., vol. 21, no. 25, pp. 31006-31011, Dec. 2013.

[13] T. Q. Wang, Y. A. Sekercioglu, and J. Armstrong, "Analysis of an optical wireless receiver using a hemispherical lens with application in MIMO visible light communications," J. Lightw. Technol., vol. 31, no. 11, pp. 1744-1754, Jun. 2013.

[14] A. Burton, H. L. Minh, Z. Ghassemlooy, E. Bentley, and C. Botella, "Experimental demonstration of $50-\mathrm{Mb} / \mathrm{s}$ visible light communications using 4 by 4 MIMO," IEEE Photon. Technol. Lett., vol. 26, no. 9, pp. 945-948, May 2014.

[15] P. E. Allen and D. R. Holberg, CMOS Analog Circuit Design. Oxford, U.K.: Oxford Univ. Press, 2012.

[16] F. Tavernier and M. Steyaert, High-Speed Optical Receivers with Integrated Photodiode in Nanoscale CMOS. Berlin, Germany: SpringerVerlag, 2011.

[17] K. Szczerba et al., "Comparison of intersymbol interference power penalties for OOK and 4-PAM in short-range optical links," J. Lightw. Technol., vol. 31, no. 22, pp. 3525-3534, Nov. 2013.

[18] J. R. Barry, Wireless Infrared Communications. Boston, MA, USA: Kluwer, 1994.

[19] J. M. Kahn and J. R. Barry, "Wireless infrared communications," Proc. IEEE, vol. 85, no. 2, pp. 265-298, Feb. 1997.

[20] J. Armstrong and B. J. C. Schmidt, "Comparison of asymmetrically clipped optical OFDM and DC-biased optical OFDM in AWGN," IEEE Commun. Lett., vol. 12, no. 5, pp. 343-345, May 2008.

[21] S. Dimitrov, S. Sinanovic, and H. Haas, "Signal shaping and modulation for optical wireless communication," J. Lightw. Technol., vol. 30, no. 9, pp. 1319-1328, May 2012.

[22] S. Randel, F. Breyer, S. C. J. Lee, and J. W. Walewski, "Advanced modulation schemes for short-range optical communications," IEEE J. Sel. Topics Quantum Electron., vol. 16, no. 5, pp. 1280-1289, Sep./Oct. 2010.

[23] S. D. Perli, N. Ahmed, and D. Katabi, "PixNet: Interference-free wireless links using LCD-camera pairs," presented at the 16th Annual Int. Conf. Mobile Computing Networking, Chicago, IL, USA, 2010.

[24] J. M. Kahn et al., "Imaging diversity receivers for high-speed infrared wireless communication," IEEE Commun. Mag., vol. 36, no. 12, pp. 88-94, Dec. 1998.

[25] Z. Lubin et al., "High data rate multiple input multiple output (MIMO) optical wireless communications using white LED lighting," IEEE J. Sel. Areas Commun., vol. 27, no. 9, pp. 1654-1662, Dec. 2009.

[26] P. Djahani and J. M. Kahn, "Analysis of infrared wireless links employing multibeam transmitters and imaging diversity receivers," IEEE Trans. Commun., vol. 48, no. 12, pp. 2077-2088, Dec. 2000.

[27] C. Lili, L. Zhiqun, W. Zhigong, L. Wei, and Z. Li, "A 10-Gb/s CMOS differential transimpedance amplifier for parallel optical receiver," in Proc. ISSSE, 2010, pp. 1-4.

[28] T. Shimotori, K. Maekita, T. Maruyama, and K. Iiyama, "Characterization of APDs fabricated by $0.18 \backslash \mu \mathrm{m}$ CMOS in blue wavelength region," in Proc. 17th OECC, 2012, pp. 509-510.

[29] S. Radovanovic, A.-J. Annema, and B. Nauta, High-Speed Photodiodes in Standard CMOS Technology. Berlin, Germany: Springer-Verlag, 2008.

[30] M.-J. Lee and W.-Y. Choi, "Area-dependent photodetection frequency response characterization of silicon avalanche photodetectors fabricated with standard CMOS technology," IEEE Trans. Electron Devices, vol. 60, no. 3, pp. 998-1004, Mar. 2013.

[31] S. Ray, M. M. Hella, M. M. Hossain, P. Zarkesh-Ha, and M. M. Hayat, "Speed optimized large area avalanche photodetector in standard CMOS technology for visible light communication," in Proc. IEEE SENSORS, 2014, pp. 2147-2150.

[32] K. Schneider and H. Zimmermann, Highly Sensitive Optical Receivers, vol. 23. Berlin, Germany: Springer-Vesrlag, 2006.

[33] Forward Error Correction for High Bit-Rate DWDM Submarine Systems, ITU, Geneva, Switzerland, 2013.

Authors' photographs and biographies not available at the time of publication. 